\title{
0 acesso de homens a diagnóstico e tratamento de doenças sexualmente transmissíveis na perspectiva multidimensional e relacional da vulnerabilidade
}

Neide Emy Kurokawa e Silva ${ }^{1}$ Leyla Gomes Sancho ${ }^{2}$

\section{Homens e atenção às doenças sexualmente transmissíveis}

Discute-se o acesso dos homens ao diagnóstico e tratamento de doenças sexualmente transmissíveis (DST), problematizando as referências clássicas sobre acesso, que tratam a questão sob o binômio demanda e oferta, e propondo compreender o fenômeno por meio do aporte conceitual da vulnerabilidade.

De doenças incuráveis e motivo de segregação social no passado, hoje, grande parte das DST são curáveis, com recurso a tratamentos relativamente simples. No entanto, ainda constituem um problema de Saúde Pública, não só no Brasil, mas, também, em todo o mundo.

Não obstante as iniciativas para mudar o panorama das DST no país, o Ministério da Saúde reconhece que

as diretrizes para diagnóstico e tratamento precoces, incluindo as parcerias sexuais, são pouco conhecidas ou implementadas pelo sistema de saúde. Não existe disponibilidade contínua de medicamentos padronizados para portadores de DST, bem como de preservativos. (Brasil, 2006, p.13)

A despeito da indicação do diagnóstico e tratamento sindrômico, a fim de otimizar a atenção, observa-se uma ênfase no diagnóstico etiológico, e o manejo sindrômico é pouco conhecido pelos profissionais de saúde.

Outra situação emblemática é a prevalência de sífilis congênita, não obstante as políticas públicas, as quais incluem um rol de ações diferenciadas de vigilância e prevenção, assistência pré-natal e ao recém-nascido (Victora et al., 2011; Ramos Júnior et al., 2007; Walker, Walker, 2007; Rodrigues, Guimarães, 2004); e, no tocante à melhoria do acesso aos serviços de saúde e ao tratamento, são recomendados desde investimentos no sistema de notificação até a capacitação de profissionais e a qualificação do atendimento (Saraceni et al., 2005).

Somando-se ao panorama esboçado, são apontadas distinções entre homens e mulheres no acesso e uso de serviços (Gomes, Nascimento, Araujo, 2007;

Figueiredo, 2005), mesmo levando em conta as inflexões no modelo assistencial, como as propostas pelo Sistema Único de Saúde, de atenção integral à saúde ou, 
mais recentemente, da Política Nacional de Atenção à Saúde do Homem (Brasil, 2009). Isso leva a crer que o modo de buscar o cuidado e a atenção às demandas dos homens, em relação às queixas sugestivas de DST, também guardam especificidades, quando comparadas às das mulheres. Ou seja, os homens não usufruem de chances semelhantes em nenhum momento do seu ciclo de vida, inclusive os parceiros das gestantes com diagnóstico de DST (Araújo et al., 2008).

Ainda que, com alguns entraves, as mulheres, ao menos aquelas que realizam o pré-natal, têm acesso ao diagnóstico e tratamento de DST. Se, por um lado, a maior parte das DST nas mulheres é assintomática (o que lhe conferiria maior suscetibilidade a agravos decorrentes dessas doenças), por outro, é obrigatório o oferecimento de exames de sífilis e HIV e o tratamento, quando for o caso, durante o pré-natal, visando reduzir a transmissão vertical.

Não dispondo de uma inserção específica nos serviços de saúde, dentre as barreiras programáticas que afetam os homens, são citadas as dificuldades para marcar uma consulta em serviço de saúde, além da restrita oferta de horários e dias de atendimento, compatíveis com as necessidades dos homens. Somada a essas barreiras, na prática, ainda paira certa indefinição quanto às responsabilidades da atenção primária no acolhimento das demandas de DST, que, mormente, são referidas a serviços especializados, independentemente da complexidade do caso (Brasil, 2006; Araújo, Leitão, 2005). Embora prevista nas diretrizes da Atenção Básica, nem sempre esse nível de atenção tem conseguido incorporar as demandas de DST em homens.

Vale frisar, ainda, que muitos homens nem chegam a buscar cuidados nos serviços de saúde, seja por estarem assintomáticos, seja por procurarem tratamentos alternativos, com remédios caseiros ou, então, "prescritos" por amigos ou nos balcões das farmácias. Ao lado das consequências mórbidas, deve ser ressaltada a presença de outras dimensões das DST, relativas à construção do imaginário sobre essas doenças, sua manipulação social e desdobramentos psicossociais, como apresentados nos trabalhos de Sontag (1989), acerca da aids e suas metáforas, ou de Carrara (1996), que trata do caráter totêmico das chamadas doenças venéreas e dos complexos processos sociais envolvidos na luta contra a sífilis.

Ainda no plano simbólico, mesmo que controverso, há o imaginário de que homem não gosta ou não valoriza o cuidado com sua saúde (Figueiredo, 2005) ou, mesmo, a resistência na utilização do preservativo (Matos, Veiga, Reis, 2009; Galvão et al., 2002).

Depreende-se, do exposto, que há um conjunto de investimentos visando o controle das DST, mas que ainda não alcançaram o impacto desejado no quadro epidemiológico, a exemplo da sífilis congênita. Como se explica essa lacuna entre investimentos e resultados? No caso dos homens, em especial, seria pertinente explorar o problema como uma questão de acesso aos serviços? A seguir, discutem-se, brevemente, as principais noções de acesso presentes na literatura, levantando suas limitações para a compreensão das possibilidades e limites no diagnóstico e tratamento de DST na população masculina.

\section{Para além da demanda e da oferta: o acesso em pauta}

Uma aproximação inicial à noção de acesso pode ser feita a partir dos seus descritores em Ciências da Saúde (DeCS - Terminologia em Saúde) e suas respectivas definições:

1) Acesso aos serviços de saúde: Possibilidade de os indivíduos adentrarem e utilizarem os serviços de atenção à saúde, com vistas à resolução de problemas que afetem a saúde. Dentre os fatores que influem nesta possibilidade, incluem-se considerações geográficas, arquitetônicas, de transporte, financeiras, entre outras.

2) Qualidade, acesso e avaliação da assistência à saúde: Conceito que se preocupa com todos os aspectos da qualidade, acessibilidade e avaliação de cuidados de saúde e entrega de cuidados de saúde.

3) Equidade ao acesso: Possibilidade do sistema de saúde oferecer alternativas para os indivíduos que mais têm dificuldades de adentrarem e se utilizarem dos serviços de saúde oferecidos, numa região territorialmente delimitada. Cada grupo, estrato social ou região apresenta problemas específicos, diferenças no modo de viver, de adoecer, de acessar os serviços de atenção à saúde e satisfazer suas 
necessidades de vida. Tais diferenças devem ser consideradas para se oferecer mais a quem mais precisa, diminuindo as desigualdades existentes.

Girando em torno dessas definições, a temática sobre o acesso, sobretudo nas áreas de planejamento e avaliação de serviços de saúde, vem sendo explorada privilegiadamente por referência às dimensões da demanda e oferta, da utilização e da capacidade de organização e respostas dos serviços (Travassos, Martins, 2004), tendo como eixo estruturante as ideias de consumo e de consumidores de serviços. Nesses termos, o diagnóstico e tratamento de DST, dos homens, estariam associados a uma questão de "logística" nos serviços de saúde, tais como horários mais flexíveis ou a existência de profissionais para atendê-los.

Uma vertente, menos explorada, focaliza as discussões sobre o acesso, sob o prisma do direito à atenção ou dos seus aspectos simbólicos, ressaltando o conceito de representações sociais (Jesus, Assis, 2010; Giovanella, Fleury, 1995), cujo mote é o de que o conhecimento das concepções sobre saúde e doença da população pode subsidiar diferentes modos de intervenções, visando garantir a entrada do usuário no sistema de saúde. Ainda assim, as preocupações práticas e teóricas sobre o acesso concentram-se na formulação de estratégias visando responder, separadamente, à oferta de serviços e às demandas dos usuários - levando a indagar, por exemplo, se a simples ciência do direito de ser atendido garantiria que os homens procurassem os serviços de saúde frente a sinais/sintomas de DST ou, mesmo, sobre o caráter instrumental do "pré-natal" do homem, quando restrito ao controle da sífilis congênita.

Ainda que alcançando diferentes perspectivas, as abordagens sobre o acesso à saúde seguem a racionalidade científica moderna de segmentação e naturalização dos fenômenos, ignorando, por vezes, a complexidade da sua produção e de suas interações, no caso, entre demanda e oferta, comum nos estudos oriundos do planejamento em saúde e inspirados nas abordagens teóricas da economia política e neoclássica (Pinheiro et al., 2005).

O tratamento isolado dos elementos que compõem o acesso resulta na fragmentação, tanto da identificação dos problemas, quanto das proposições para sua solução: se é tomado como um problema da prática dos profissionais de saúde, a resposta é a proposição de capacitações e treinamentos; se é a incompatibilidade entre o horário do trabalhador e do serviço, a solução é a flexibilização dos horários de funcionamento; se é a captação de parceiros de gestantes com diagnóstico de sífilis, a saída é o prénatal do homem.

Sem caber aqui uma crítica aos problemas identificados e às soluções produzidas, o questionamento recai sobre a lógica que informa os modos de apreensão e respostas aos obstáculos que se interpõem à saúde. Mesmo que buscando superar a ideia de "contrário de doença" (Ayres, 2007; Camargo Júnior, 2004), o conceito de saúde que remonta as proposições da Declaração de Alma Ata, tomado como "completo bem estar físico, mental e social", tem ensejado abordagens compartimentalizadas, por referência às dimensões físicas e mentais e sociais, isoladamente.

A "imagem idílica" de que seria possível um ajuste perfeito entre a emergência de um problema, a formulação de uma demanda e as respostas dos serviços de saúde, por prioridades (Camargo Júnior, 2005, p.92), reporta a uma premissa de que os problemas de saúde são “objetos dados, que se oferecem passivamente à observação, bastando serem corretamente identificados pelas técnicas adequadas".

Ocorre que, entre a identificação do problema e a motivação para buscarem algum tipo de atenção; entre as barreiras encontradas pelos homens para acessar os serviços de saúde e as dificuldades no acolhimento de suas demandas; entre as proposições das políticas e protocolos e as possibilidades práticas na sua implementação, perpassa uma complexa e dinâmica rede envolvendo diferentes interações entre atores, protocolos de tratamento, fluxos, políticas, normas culturais e sociais.

Para fazer face a essas preocupações, é preciso mais que a escolha de um modelo explicativo para o problema do acesso, posto que este nem capta as especificidades de ser homem nem a dinâmica das relações que estabelece com os diferentes atores em diferentes contextos - familiares, dos serviços de saúde ou das normas culturais e sociais mais amplas. 
Nesse sentido, parece oportuno recorrer a um quadro compreensivo que incorpore e sintetize os diferentes contextos que podem facilitar ou limitar o acesso dos homens aos serviços de saúde, notadamente para o diagnóstico e tratamento de DST.

\section{Das barreiras à vulnerabilidade no acesso dos homens ao diagnóstico e tratamento de DST}

Como já mencionado, ainda que sugerindo certa articulação entre diferentes dimensões, as abordagens sobre acesso são geralmente traduzidas em termos de barreiras à atenção à saúde.

A proposição do adensamento do conceito de acesso aos serviços de saúde considera o deslocamento do sentido de barreira, tomada como uma entidade ou como conjunto de fatores tratados isoladamente, para a construção de sínteses, possível por meio do quadro da vulnerabilidade (Ayres et al., 2009, 2003).

É relativamente recente a incorporação do termo vulnerabilidade no campo da saúde, tendo como marco inicial os debates e estudos em torno da epidemia de aids na década de 1980 e da crítica ao conceito epidemiológico de risco, que se mostrava pouco produtivo tanto na compreensão do fenômeno quanto no controle da epidemia.

Sem adentrar no detalhamento histórico da apreensão do conceito, a principal crítica residia no fato de que as categorias analíticas que visavam objetivar o fenômeno da aids não conseguiam apreender a complexidade da vida dos sujeitos. O isolamento dos fatores de risco não logrou êxito quanto ao controle da epidemia e, além disso, acabou fomentando a segregação de determinados indivíduos e grupos, suscetibilizando-os a situações de estigma e discriminação, sobretudo quando do trânsito dos fatores de risco para os chamados grupos e comportamentos de risco.

Se os estudos sobre o risco seguem uma racionalidade analítica na qual os fenômenos são segmentados, isolados, discriminados, aqueles pautados na vulnerabilidade coadunam-se com uma racionalidade sintética, que privilegia a construção de significados e a agregação de elementos diversos que contribuem para que os diferentes fenômenos em estudo sejam compreendidos como uma totalidade dinâmica e complexa.

Estudar o acesso dos homens ao diagnóstico e tratamento de DST sob a perspectiva da vulnerabilidade implica não apenas ater-se à sua dimensão programática, como a oferta, organização e qualidade dos serviços, mas, também, incorporar à atenção aspectos individuais como valores, crenças, atitudes envolvidas, bem como as normas sociais e culturais com as quais interagem.

Talvez mais do que ampliar o alcance de questões a serem consideradas em relação ao acesso, o referencial da vulnerabilidade se destaca pelo fato de fomentar um tipo de raciocínio que não busca o estabelecimento de relações de causalidade, mas o exercício de um olhar que se movimenta entre a interpretação e a compreensão dos diversos contextos, a fim de informar a ação.

Nesse sentido, a construção da demanda dos homens, frente à suspeita de DST, pode associar-se ao imaginário acerca dessas doenças, motivando-o, ou não, a procurarem um serviço de saúde, atentandose para as distinções simbólicas, entre elas: enquanto a aids tem sido associada à homossexualidade, a sífilis, por exemplo, pode ser um sinal de virilidade para os homens (Carrara, 1994). Por sua vez, referida distinção pode não proceder ou, mesmo, articular-se ao fato de que admitir a suspeita de uma DST pode transparecer ou visibilizar eventual infidelidade conjugal, o que interferiria sobremaneira na busca por atenção ao problema.

A atenção primária é a porta de entrada principal e ordenadora dos demais níveis de complexidade para o Sistema Único de Saúde - SUS, tendo como estratégia privilegiada a atenção por meio de equipes de saúde da família. Mas, se a suspeita de DST estiver associada a práticas que merecem ser guardadas em segredo, como compartilhá-la com os agentes comunitários de saúde, geralmente responsáveis por intermediar a marcação de consultas, mas que são pessoas de sua convivência ou conhecidos do seu meio?

O Decreto no. 7.508/2011 atenuaria esse impasse ao preconizar outras portas de entrada para o SUS, não restritas à atenção primária: os serviços de urgência e emergência e os especiais de acesso aberto, no caso, os serviços especializados em DST e aids. 
Do ponto de vista normativo, o conteúdo do referido Decreto propõe o acolhimento da população em qualquer um dos pontos do sistema de saúde, flexibilizando a ideia de que essa iniciativa caberia apenas à atenção primária. Essa prerrogativa pode ser profícua para o caso dos homens com suspeita de DST, pois ampliaria suas referências de saúde, para além do posto de saúde próximo à sua residência ou, mesmo, os balcões de farmácias.

A propósito, as farmácias mereceriam especial atenção das políticas públicas e práticas dos serviços de saúde, no sentido de não apenas coibir ética e tecnicamente as prescrições dos balconistas, mas de explorar suas potencialidades, tanto na prevenção quanto no encaminhamento para um tratamento adequado das DST, como já apontado em estudos como os de Naves et al. (2008) e Rosso Neto e Galato (2011).

A possibilidade de acolhimento das demandas em qualquer ponto do sistema de saúde e a abordagem sindrômica das DST poderiam constituir-se em associação bastante fecunda no tratamento dos homens, entretanto, como já apontado, não se trata de uma rotina observada no cotidiano. Muitas vezes, nem mesmo os parceiros de gestantes com diagnóstico de DST são acolhidos pelos próprios médicos que as acompanham no pré-natal.

Antes de subsumir esse fato a uma questão de treinamento e capacitação dos profissionais de saúde, caberia também indagar sobre os motivos pelos quais não se conta nem com o acolhimento nem com o tratamento sindrômico. O que pensam os médicos sobre esse tipo de tratamento, comparativamente à abordagem etiológica? Que tipo de resistências os médicos ginecologistas, geralmente responsáveis por acompanhamento de gestantes, podem apresentar no atendimento de homens? Essa resistência se agrava quando se trata de médicas? Será que o tempo disponibilizado para as consultas permite abordar os parceiros?

É certo que a não-implementação das recomendações ministeriais, que preconizam o diagnóstico e tratamento sindrômicos, não se restringe aos consultórios de pré-natal. Caberia aprofundar as indagações sobre os motivos dessa resistência, sobre como os serviços e profissionais de saúde apreendem referidas recomendações; sobre os valores, recursos, habilidades, capacidades e limites envolvidos nos diferentes níveis e tipos de atenção, já que não é raro, por exemplo, presenciar situações em que homens com suspeita de DST não são atendidos em prontos-socorros porque suas queixas não se enquadram como emergência/urgência.

São muitos os aspectos a serem considerados ao se pretender tomar a questão do acesso na perspectiva da vulnerabilidade, superando as leituras compartimentalizadas acerca do processo de construção da demanda e da atenção nos serviços de saúde.

Perante o problema apresentado - do acesso dos homens ao diagnóstico e tratamento de DST -, o conceito de vulnerabilidade visa contribuir para um adensamento da noção de acesso no campo da saúde, tomando-o a partir de diferentes contextos que interfeririam na trajetória dos homens, desde a sua identificação como um problema de saúde até a busca por cuidado e o seu acolhimento pelos serviços de saúde.

A compreensão do acesso dos homens ao diagnóstico e tratamento de DST, sob a perspectiva da vulnerabilidade, pode subsidiar ações de planejamento e avaliação de programas e serviços de saúde atinentes ao controle das DST, bem como o desenvolvimento de ações e instrumentos tecnológicos específicos, delineando um quadro que expresse a dinamicidade dos diferentes contextos que modelam desde o imaginário sobre as DST no universo masculino até a construção de políticas e diretrizes para essa atenção. Vale alertar, contudo, que a proposição conceitual da vulnerabilidade não pode confundirse com, ou, mesmo, substituir uma teoria ou método de planejamento e gestão, como lembram Ayres et al. (2003).

Mais que apontar questões para a área específica da saúde, o enfoque da vulnerabilidade pode cruzar os seus muros, apreendendo outras instâncias que podem se constituir em barreiras ao acesso dos homens na atenção às DST, evidenciando as articulações interinstitucionais envolvidas no problema, como o investimento em ambulatórios de indústrias e empresas, especialmente aquelas que abrigam majoritariamente trabalhadores do sexo masculino.

Outra contribuição que pode ter efeitos práticos, embora não imediatos, diz respeito à identificação de aspectos estruturais e sociais mais amplos que repercutiriam no acesso ao diagnóstico e tratamento 
de DST, a exemplo das desigualdades de gênero. Ainda que não surta efeitos a curto prazo, a assunção dessa realidade é fundamental na formulação das políticas e iniciativas visando o controle das DST.

No plano do conhecimento, é clara a exigência de investimentos interdisciplinares que façam face à complexidade da Saúde Coletiva - e as análises baseadas na vulnerabilidade podem propiciar esse movimento. Além da oportunidade do "diálogo" entre diferentes disciplinas, o quadro da vulnerabilidade permite, ainda, "resgatar a dignidade epistemológica das relações partes-todo na apreensão teórica dos fenômenos da saúde coletiva" (Ayres et al., 2003, p.137). Ou seja, ao propor a integração entre disciplinas biomédicas e das ciências sociais e humanas, e entre as linguagens abstratas das tecnociências e os chamados conhecimentos leigos, ressaltam-se as dimensões éticas, políticas e relacionais das práticas de saúde (Ayres et al., 2003).

Das inúmeras imagens que o quadro da vulnerabilidade pode ancorar, a de "carta de navegação" parece ir ao encontro da proposição de compreender o acesso dos homens ao diagnóstico e tratamento de doenças sexualmente transmissíveis:

Como a carta de navegação, processos de planejamento e avaliação não determinam para onde se vai navegar, não nos dão o controle do que vai acontecer na travessia, nem substituem o ato mesmo de navegar. Mas eles podem nos ajudar a definir em que direção queremos seguir, perceber o que vai acontecendo pelo caminho e o que podemos e queremos fazer diante desses acontecimentos. (Ayres, 2009, p.410)

\section{Colaboradores}

Neide Emy Kurokawa e Silva foi responsável pela elaboração do artigo, e Leyla Gomes Sancho participou da concepção, da discussão e da revisão do manuscrito.

\section{Referências}

ARAUJO, M.A.L. et al. Análise da qualidade dos registros nos prontuários de gestantes com exame de VDRL reagente. Rev. APS, v.11, n.1, p.4-9, 2008.

ARAÚJO, M.A.L.; LEITÃO, G.C.M. Acesso à consulta a portadores de doenças sexualmente transmissíveis: experiências de homens em uma unidade de saúde de Fortaleza, Ceará, Brasil. Cad. Saude Publica, v.21, n.2, p.396-403, 2005.

AYRES, J.R.C.M. Uma concepção hermenêutica de saúde. Physis, v.17, n.1, p.43-61, 2007.

AYRES, J.R.C.M. et al. Risco, vulnerabilidade e práticas de prevenção e promoção da saúde. In: CAMPOS, G.W.S. et al. (Orgs.). Tratado de saúde coletiva. São Paulo: Hucitec, 2009. p.375-418.

. O conceito de vulnerabilidade e as práticas de saúde: novas perspectivas e desafios. In: CZERESNIA, D.; FREITAS C.M. (Orgs.). Promoção da saúde: conceitos, reflexões, tendências. Rio de Janeiro: Editora Fiocruz, 2003. p.117-40. 
BRASIL. Ministério da Saúde. Secretaria de Atenção à Saúde. Departamento de Ações Programáticas e Estratégicas. Plano de Ação Nacional 2009-2011 da Política Nacional de Atenção Integral à Saúde do Homem. Brasília: MS, 2009.

Ministério da Saúde. Secretaria de Vigilância em Saúde. Programa Nacional de $\overline{\mathrm{DST} / \mathrm{Ai}}$ ds. Manual de controle das doenças sexualmente transmissíveis. 4. ed. Brasília: MS, 2006. (Série A, Normas e Manuais Técnicos).

CAMARGO JÚNIOR, K. Das necessidades em saúde à demanda socialmente constituída. In: PINHEIRO, R.; MATTOS, R.A. (Orgs.). Construção social da demanda: direito à saúde, trabalho em equipe, participação em espaços públicos. Rio de Janeiro: IMS/UERJ, Cepesc, Abrasco, 2005. p.91-101.

Epistemologia numa hora dessas? (Os limites do cuidado). In: PINHEIRO, R.; MATTOS, R.A. (Orgs.). Cuidado: as fronteiras da integralidade. Rio de Janeiro: Hucitec/ Abrasco, 2004. p.157-70.

CARRARA, S. A Aids e a história das doenças venéreas no Brasil. In: LOYOLA, M.A. (Org.). Aids e sexualidade. Rio de Janeiro: Relumé-Dumará/UERJ, 1994. p.73-108.

Tributo a Vênus: a luta contra a sífilis no Brasil. Rio de Janeiro: Fiocruz, 1996.

FIGUEIREDO, W.S. Assistência à saúde dos homens: um desafio para os serviços de atenção primária. Cienc. Saude Colet., v.10, n.1, p.105-9, 2005.

GALVÃO, M. et al. Razões do não uso do preservativo masculino entre pacientes com infecção ou não pelo HIV. DST J. Bras. Doenças Sex. Transm., v.25, n.1, p.25-30, 2002.

GIOVANELLA, L.; FLEURY, S. Universalidade da atenção à saúde: acesso como categoria de análise. In: EIBENSCHUTZ, C. (Org.). Política de saúde: o público e o privado. Rio de Janeiro: Fiocruz, 1995. p.177-98.

GOMES, R.; NASCIMENTO, E.F.; ARAUJO, F.C. Por que os homens buscam menos os serviços de saúde do que as mulheres? As explicações de homens com baixa escolaridade e homens com ensino superior. Cad. Saude Publica, v.23, n.3, p.565-74, 2007.

JESUS, S.L.A.; ASSIS, M.M.A. Revisão sistemática sobre o conceito de acesso nos serviços de saúde: contribuições do planejamento. Cienc. Saude Colet., v.15, n.1, p.161-70, 2010.

MATOS, E.B.; VEIGA, R.T.; REIS, Z.S.N. Intenção de uso de preservativo masculino entre jovens estudantes de Belo Horizonte: um alerta aos ginecologistas. Rev. Bras. Ginecol. Obstet., v.31, n.11, p.574-80, 2009.

NAVES, J.O.S. et al. Práticas de atendimento a DST nas farmácias do Distrito Federal, Brasil: um estudo de intervenção. Cad. Saude Publica, v.24, n.3, p.577-86, 2008.

PINHEIRO, R. et al. Demanda em saúde e direito à saúde: liberdade ou necessidade? Algumas considerações sobre os nexos constituintes das práticas de Integralidade. In: PINHEIRO, R.; MATTOS, R.A. (Orgs.). Construção social da demanda: direito à saúde, trabalho em equipe, participação em espaços públicos. Rio de Janeiro: IMS/UERJ, Cepesc, Abrasco, 2005. p.11-27.

RAMOS JÚNIOR, N.A. et al. Control of mother-to-child transmission of infectious diseases in Brazil: progress in HIV/AIDS and failure in congenital syphilis. Cad. Saude Publica, v.233, supl.3, p.S370-8, 2007.

RODRIGUES, C.S.; GUIMARÃES, M.D.C. Grupo Nacional de Estudo sobre Sífilis Congênita. Positividade para sífilis em puérperas: ainda um desafio para o Brasil. Rev. Panam. Salud Publica, v.16, n.3, p.168-75, 2004.

ROSSO NETO, C.; GALATO, D. A contribuição dos estabelecimentos farmacêuticos na prevenção e no manejo das DST: um estudo qualitativo em uma cidade do sul do Brasil. DST J. Bras. Doenças Sex. Transm., v.23, n.2, p.120-5, 2011. 
O ACESSO DE HOMENS A DIAGNÓSTICO E TRATAMENTO ...

SARACENI, V. et al. Mortalidade perinatal por sífilis congênita: indicador de qualidade da atenção à mulher e à criança. Cad. Saude Publica, v.21, n.4, p.1244-50, 2005.

SONTAG, S. Aids e suas metáforas. São Paulo: Companhia das Letras, 1989.

TRAVASSOS, C.; MARTINS, M. Uma revisão sobre os conceitos de acesso e utilização de serviços de saúde. Cad. Saude Publica, v.20, supl.2, p.S190-S8, 2004.

VíCTORA, C.G. et al. Maternal and child health in Brazil: progress and challenges. Lancet, v.377, n.9780, p.1863-76, 2011.

WALKER, J.G.; WALKER, D.G. Congenital syphilis: a continuing but neglected problem. Semin. Fetal Neonatal Med., v.12, n.3, p.198-206, 2007. 
Discute-se a noção de acesso de homens à atenção à saúde, especificamente para diagnóstico e tratamento de doenças sexualmente transmissíveis - DST. Considerando que o acesso à saúde está imbricado em uma rede de interações e contextos sociais que ultrapassa o binômio demanda-oferta, postula-se a pertinência de desenvolver o tema sob o conceito multidimensional e dinâmico de vulnerabilidade.

Palavras-chave: Acesso aos serviços de saúde. Identidade de gênero. Homens. Doenças sexualmente transmissíveis. Vulnerabilidade.

Men's health care access to diagnosis and treatment of sexually transmitted diseases in a multidimensional and relational concept of vulnerability

This paper discusses the notion of men's access to health care, specifically for diagnosis and treatment of sexually transmitted diseases - STD. As access to health care is entangled in a network of interactions and social contexts beyond the demand-supply binomial, the paper postulates the relevance of developing this theme under the multidimensional and dynamic concept of vulnerability.

Keywords: Health services accessibility. Gender identity. Men. Sexually transmitted diseases. Vulnerability.

\section{El acceso de los hombres para el diagnóstico y tratamiento de enfermedades de transmisión sexual en la perspectiva multidimensional y relacional de la vulnerabilidad}

Se discute la noción de acceso a los servicios de salud, específicamente para diagnóstico y tratamiento de enfermedades transmitidas sexualmente - ETS, en el caso de hombres. Considerando que el acceso a la salud está imbricado en una red de interacciones y contextos sociales que sobrepasa el binomio demanda-oferta, se postula la pertinencia de desarrollar el tema según el concepto multidimensional y dinámico de vulnerabilidad.

Palabras clave: Accesibilidad a los servicios de salud. Identidad de género. Hombres. Enfermedades transmitidas sexualmente. Vulnerabilidade. 
